\title{
Aucun, évolution diachronique
}

\author{
Asma Dhoukar \\ MoDyCo, Université Paris Nanterre, 200 avenue de la République 92001 Nanterre Cedex, France \\ asma141982@yahoo.fr
}

Résumé. Ce papier se propose de décrire l'évolution diachronique de l'indéfini aucun. Nous évoquerons d'abord son évolution sémantique: valeur positive et valeur négative. Il sera question ensuite de son évolution catégorielle : déterminant indéfini et pronom indéfini. Nous observerons également son évolution morphologique: variation en genre (aucun/aucune) et en nombre (aucun/aucuns). Nous déterminerons finalement son évolution syntaxique: cas de postposition (sentiment aucun), cas d'insertion d'adjectifs ou d'indéfinis dans le groupe aucun $+\mathrm{N}$ $($ aucun + beau/autre $+\mathrm{N})$ et la construction directe/indirecte de l'épithète ou de l'indéfini que l'on peut joindre à aucun (aucun sérieux/autre vs aucun de sérieux/d'autre).

\begin{abstract}
Aucun, diachronic evolution. This paper is used to describe the diachronic evolution of the indefinite aucun. We will first talk about its semantic evolution : positive value and negative value. We will treat later its categorical evolution : indefinite determiner and indefinite pronoun. Also, we will observe its morphological evolution: variation in gender (aucun, aucune) and in number (aucun, aucuns). Finally, we will determine its syntactic evolution: case of "postposition» (sentiment aucun), case of the insertion of adjective or indefinite determiner or pronoun in the group aucun $+\mathrm{N}$ (aucun sérieux/autre vs aucun de sérieux/d'autre) and the direct/indirect construction of the adjective or the indefinite determiner or pronoun that we can join to aucun (aucun sérieux/autre vs aucun de sérieux/d'autre).
\end{abstract}

\section{Introduction}

L'indéfini aucun a fait l'objet, dans les grammaires, d'études qui restent limitées à des généralités sans approfondir certains points spécifiques. Ce papier se propose donc de décrire l'évolution diachronique de l'indéfini aucun. C'est une étude primordiale et nécessaire dans la mesure où, d'après Pierrard (1998: 38), de «nombreux linguistes (cf. Traugott \& Heine, 1991 ; Hopper \& Traugott, 1993 ; Bat-Zeev, 1994) affirment aujourd'hui «que l'on ne peut comprendre le fonctionnement des éléments grammaticaux avant d'avoir, au préalable, étudié et analysé les processus diachroniques que ces éléments ont dû suivre » (Bat-Zeev, 1994: 114). Pour essayer de comprendre le fonctionnement de l'indéfini aucun, nous évoquerons d'abord son évolution sémantique : variation entre valeur positive et valeur négative. Il sera question ensuite de son évolution catégorielle : il peut fonctionner comme déterminant indéfini ou pronom indéfini. Nous observerons également son évolution morphologique: variation en genre (masculin/féminin) et en nombre 
(singulier/pluriel). Nous déterminerons finalement son évolution syntaxique: cas de postposition $(\mathrm{N}+$ aucun $)$, cas d'insertion d'adjectifs ou d'indéfinis dans le groupe aucun + $\mathrm{N}$ (aucun + adjectif/indéfini $+\mathrm{N})$ et la construction directe/indirecte de l'épithète ou de l'indéfini que l'on peut joindre à aucun (aucun + adjectif/indéfini vs aucun + de + adjectif/indéfini).

Pour mener à bien cette étude, nous avons interrogé un corpus suivant une perspective diachronique allant du $\mathrm{XII}^{\mathrm{e}}$ siècle jusqu'au $\mathrm{XX}^{\mathrm{e}}$ siècle inclus. Il est formé de trois bases de données $^{1}$ qui se recouvrent en partie à savoir la Base du Français Médiéval ${ }^{2}$ (du XII ${ }^{\mathrm{e}}$ jusqu'au début du XIV ${ }^{\mathrm{e}}$ siècle), le Dictionnaire du Moyen Français ${ }^{3}$ (du XIV XI $^{\mathrm{e}}$ jusqu'au début du $\mathrm{XVI}^{\mathrm{e}}$ siècle) et Frantext ${ }^{4}$ pour les siècles suivants (du $\mathrm{XVI}^{\mathrm{e}}$ jusqu'au $\mathrm{XX}^{\mathrm{e}}$ siècle).

\section{Aucun, évolution sémantique}

Jespersen (1971) remarque que, dans les langues, l'évolution du positif vers le négatif est très fréquente. Pour ce qui est du français, l'évolution peut s'effectuer du positif vers le négatif ou du négatif vers le positif. En ce qui concerne aucun, le consensus semble établi par les grammaires, selon lequel ce mot se spécialise dans le sens négatif au cours de l'histoire de la langue. Nous notons un certain flottement quant à l'époque où s'établit ce changement, ce qui mérite une étude pour la préciser plus nettement. Nous nous intéressons donc ici à la variation sémantique de aucun et plus particulièrement aux contextes de son emploi en tant que mot positif ou négatif. D’une manière générale et en se référant aux différentes grammaires citées dans la bibliographie, aucun présente deux valeurs bien distinctes que l'on peut résumer dans cette citation de Fournier (1998 : 233) :

«La position la plus répandue, chez les grammairiens classiques mais aussi chez les historiens de la langue, est d'opposer deux valeurs distinctes : une valeur positive, étymologique (aucun < aliquis unus [...]) mais marginale, et une valeur négative, saillante, qui s'est imposée aux dépens de la première $»$.

Spillebout (1985 : 88-89) ajoute que ces deux valeurs étaient en concurrence, la langue classique du XVII ${ }^{\mathrm{e}}$ siècle a gardé de la langue du XVI ${ }^{\mathrm{e}}$ siècle deux valeurs centrales de aucun : une valeur positive et une valeur négative « qui se retrouvent concurremment dans toutes les catégories d'emploi » :

(1) Car apres avoir entendu leurs charges, il respondit : qu'il estoit tresobeïssant fils de la saincteté du pape, et prest à combattre pour son authorité, si aucun le vouloit quereller. (Fauchet, Fleur de la maison de Charlemaigne, 1601)

(2) Vos voeux si mal reçus de l'ingrate Dorise, Qui le caresse autant comme elle vous méprise, Ne rencontreront plus aucun empêchement. (Corneille, Clitandre ou l'Innocence délivrée, 1632)

Outre cette position opposant valeur positive et négative, Fournier (1998: 233) ajoute une autre, inspirée de Damourette \& Pichon, consistant à « proposer un traitement unitaire de ces indéfinis [aucun, rien, personne...] et de postuler une valeur de base, qui s'actualiserait en un effet de sens positif ou négatif selon les contextes ». Ces indéfinis sont considérés alors comme des forclusifs ${ }^{5}$ ayant pour valeur de base « de classer ce qu'ils expriment hors du champ de ce qui est aperçu comme réel ou réalisable » (EGLF: § 2241) ou comme des termes de polarité ${ }^{6}$. Ces forclusifs, parmi lesquels figure aucun, « apparaissent dans trois contextes d'emploi qui construisent leurs trois valeurs typiques : a) en atmosphère forclusive, avec une valeur positive inférant invalidation probable, b) avec le discordantiel ne et la valeur résultante négative, c) comme premier introducteur de forclusion avec pleine valeur négative » (Fournier, 1998 : 234). 


\subsection{Aucun, en atmosphère forclusive avec une valeur positive}

L'atmosphère forclusive concernant l'indéfini aucun se trouve assurée par divers marqueurs : des propositions interrogatives directes et indirectes ; une subordonnée régie par une principale négative; la présence d'adjectif, verbe ou nom « forcluant $^{7} »$; la préposition ou la locution «forcluante » sans (que); les locutions avant (de, que), loin (de, que) ; la locution adverbiale comme si ; des constructions comparatives et des constructions hypothétiques. Ces différents cas seront illustrés, quand notre corpus le permet, par les exemples qui persistent en français moderne.

\subsubsection{Propositions interrogatives directes et indirectes}

Certaines grammaires, allant de l'ancien français jusqu'au français moderne, se mettent d'accord sur le fait que aucun a une valeur positive dans les interrogatives :

«Dans la presque totalité des œuvres littéraires, aucun n'apparaît pas dans des phrases négatives ; il n'apparaît avec ne que dans des acceptions nettement positives encore, dans des situations non thétiques : [...] négation mise en question par une interrogative ». (Buridant, $2000: 179-180$ )

«En atmosphère forclusive, aucun est glosable par «quelque/quelqu'un » et construit une valeur de singularité/pluralité indéterminée [dans une] interrogation ». (Fournier, $1998: 234)$

«AUCUN [...] [a] le sens positif [...] dans les interrogatives directes ou indirectes ». (Chevalier et al., $1964: 267$ )

L'exploration de notre corpus nous a permis de relever les deux interrogatives suivantes (interrogative directe dans (3) et interrogative indirecte dans (4)) :

(3) Pouvait-il trouver en lui-même aucun signe de vieillissement? (Druon, Les Grandes familles : $t .1,1948$ )

(4) Je ne savais pas laquelle de ces jeunes filles était Mlle Simonet, si aucune d'elles s'appelait ainsi [...]. (Proust, À la recherche du temps perdu, 1918)

\subsubsection{Subordonnée régie par une principale négative}

Fournier (1998 : 234) note que l'emploi de aucun, en français classique, avec une valeur positive est possible quand la négation porte sur le «verbe principal». C'est le cas également en français moderne : Chevalier et al. (1964: 267 ) utilisent aucun avec une valeur positive "dans une proposition subordonnée ou dans le groupe d'un verbe à l'infinitif qui dépendent d'un verbe de forme ou de sens négatif » :

(5) Et je ne pense pas qu'aucun amour puisse compenser cela. (Alain-Fournier, Correspondance avec Jacques Rivière (19051914), 1914)

\subsubsection{Adjectif, verbe ou nom « forcluant »}

Dans la grammaire moderne, nous constatons que aucun présente une valeur positive après un adjectif ${ }^{8}$ comme dans (6), un verbe ${ }^{9}$ comme dans (7) ou un nom ${ }^{10}$ comme dans (8) « de sens négatif (nier, la négation, indéniable, etc.) ou contenant une idée de négation (défendre, le doute, incertain, etc.) » (TLFi) : 
(6) Quant à Noémi, son attitude envers ces petits est un des mystères de cette femme qui semblait incapable d'en avoir aucun. (Yourcenar, Le Labyrinthe du monde: II Archives du Nord, 1977)

(7) [...] je douterais que le sieur Pereyre, avec tout son talent, pût jamais tirer d'eux aucun chant musical. (Derrida, De la grammatologie, 1967)

(8) Il y était spécifié que l'oiseau sera fait de bois... il sera posé sur deux pattes avec défense absolue d'y mettre aucun fer ou laiton... (Jeux et sports, 1967)

C'était le cas également en français classique après, selon Fournier (1998: 234), un adjectif, un verbe ou un nom « forcluant».

\subsubsection{Préposition ou locution «forcluante » sans (que)}

Selon Chevalier et al. (1964: 267) ou le TLFi, aucun est employé couramment avec une valeur positive après la préposition sans comme dans (9) ou la locution conjonctive sans que comme dans (10):

(9) Le quinquina guérit la fièvre sans que nous ayons aucun besoin de savoir ce que c'est que la fièvre. (Bernard, Principes de médecine expérimentale, 1878)

(10) [...] malheureusement elles y répondent trop facilement, sans exiger de nous presque aucun effort [...]. (Histoire générale des sciences, 1961)

\subsubsection{Locutions avant (de, que), loin (de, que)}

Aucun peut avoir une valeur positive après, comme mentionné dans le TLFi, les tours avant de (11), avant que (12) et loin de (13) :

(11) [...] mais la grande tentative d'un théâtre entièrement nouveau à laquelle je m'adonne, me prendra plusieurs années, avant de montrer aucun résultat extérieur. (Mallarmé, Correspondance : t. 2 : 1871-1879, 1879)

(12) [...] nous le vîmes se lever d'un bond et courir se perdre à vingt mètres de là dans la foule de ses compagnons, avant qu'aucun wachmann eût trouvé le loisir d'épauler à nouveau. (Ambrière, Les Grandes vacances, 1946)

(13) Dans les romans de Malraux, de Colette, de Montherlant (dont je suis loin de penser aucun mal) il n'y a que Malraux, que Colette, que Montherlant, c'est assez clair. (Gracq, En lisant, en écrivant, 1980)

Notons, comme le remarque le TLFi, que, dans ces différentes attestations, " le verbe de la subordonnée complétive, lorsqu'il est conjugué, l'est toujours au subjonctif qui permet de présenter le procès comme l'objet d'un sentiment, d'un jugement et non comme un simple fait que l'on énonce en l'actualisant $»$.

\subsubsection{Locution adverbiale comme si}

Selon Grevisse (1993 : 931), aucun, sur le modèle d'autres auxiliaires de négation, «peut encore, dans certaines conditions [entre autres les phrases introduites par comme si], s'employer avec son ancienne valeur positive (« quelque, un, n'importe quel ») » : 
(14) Un rationalisme indigne de son nom veut ignorer ces endroits souverains. Comme si la raison pouvait mépriser aucun fait d'expérience! (Barrès, La Colline inspirée, 1913)

\subsubsection{Constructions comparatives}

Aucun s'emploie avec une valeur positive dans des constructions comparatives marquant soit l'égalité par l'intermédiaire de aussi et autant; soit l'inégalité comme le notent Chevalier et al. (1964 : 267) : "AUCUN [...] [a] le sens positif [...] dans le second terme d'une comparaison d'inégalité » via mieux, moins et plus comme dans (15) :

(15) L'agitation de ces après-midi avenue Gabriel, de ces soirées électriques, était pour moi un moment de vie intense, un moment of being plus vif qu'aucun autre moment de la semaine. (Roubaud, Poésie : récit, 2000)

\subsubsection{Constructions hypothétiques}

La valeur positive de aucun se trouve favorisée surtout par l'emploi d'hypothétiques introduites par la conjonction si, comme le remarquent Chevalier et al. (1964: 267) : «AUCUN [...] [a] le sens positif [...] après [...] parfois la conjonction SI (introduisant une conditionnelle)» :

(16) [...] si la mise au pilori de l'éclair par M. Bojarski valait la séparation au coin de la rue Daunou, si aucune phrase au monde valait cette forme de Bella entrevue dans le miroir du magasin, valait le désespoir quotidien, sans remède, de notre séparation... (Giraudoux, Bella, 1926)

Pour conclure, notons que la valeur positive de aucun se trouve favorisée généralement par une atmosphère où règne le hasard, l'imprévu, où rien ne semble être sûr, une atmosphère teintée de doute corrélée, dans certains cas, à l'emploi du subjonctif ou du conditionnel marquant ainsi l'irréalité du contexte. Ces différents contextes favorisent la lecture positive de aucun grâce au non emploi de la particule de négation ne :

«Enfin, dans certains types d'environnement (phrases interrogatives, verbe ou adjectif de sens négatif, préposition sans, etc.), les quantificateurs de l'ensemble vide peuvent être remplacés par des éléments de sens positif : sans voir personne et sans voir quelqu'un peuvent s'utiliser pour décrire la même situation ». (Arrivé et al., $1986: 327)$

Mise à part cette atmosphère forclusive, aucun peut s'employer, comme le note Fournier (1998: 235), avec une valeur positive "et opérer une quantification plurielle » dans d'autres types de contextes ${ }^{11}$ ou au pluriel $^{12}$.

\subsection{Aucun, valeur négative (ne)}

En dehors de ces tours [les différents contextes, que nous venons d'évoquer, favorisant la valeur positive de aucun], "aucun ne s'emploie plus qu'accompagné d'une négation, (ou que dans une phrase de valeur négative) [ayant comme équivalents] nul ou pas un » (Le Bidois, G. \& Le Bidois, R., 1967 : 216). Certains exemples attestent la valeur négative de aucun depuis l'ancien français et plus exactement, comme mentionné par Buridant (2000 : 180 ), dès la fin du XIII" siècle «dans des textes juridiques, dont les premières chartes en langue vulgaire [et] dans des traductions respectant la lettre du texte ou ayant tendance à latiniser [...]. L'usage se répand ensuite largement dans la langue littéraire vulgaire ». Ces différents emplois, comme le notent Picoche \& Marchello-Nizia (2008: 261), s'avèrent 
rares, « ce n'est qu'à partir du $\mathrm{XV}^{\mathrm{e}} \mathrm{s}$. que, remplaçant de plus en plus souvent $n u l$ auprès de $n e$, il prend peu à peu au singulier la valeur négative qu'il a en français moderne ». Elles ajoutent (Ibid.) que «cette évolution est achevée au XVII ${ }^{\mathrm{e}}$ s. ». La valeur négative est due alors, comme le remarque Fournier (1998: 235), au discordantiel $n e$ : « la valeur résultante est négative, ne... aucun construit une quantification nulle». C'est d'ailleurs ce que mentionnent Foulet (1919), Brunot \& Bruneau (1969), Ménard (1973), Grevisse (1990 et 1993) et Littré (1873) expliquant que cette valeur négative n'est qu'un effet de « contagion » suite à l'emploi fréquent de aucun dans des phrases de modalité négative. En voici quelques citations :

«le sens négatif [de aucun] ne lui vient que par son adjonction avec la négation $n e$ ». (Littré, $1873: 240$ )

«Au moment même où quelque passait du sens dubitatif au sens affirmatif, un autre mot suivait une évolution précisément inverse : c'est aucun [...]. Dès le XII ${ }^{\mathrm{e}}$ siècle on rencontre aucun, et toujours au sens de «quelqu'un ». Le mot devient de plus en plus fréquent, sans modifier sa signification. Puis les phrases négatives où il se glisse déteignent sur lui. Il finira au $\mathrm{XV}^{\mathrm{e}}$ siècle par prendre un sens nettement négatif, qu'il a encore aujourd'hui. On voit que c'est tout à fait l'histoire de quelque, mais vue à rebours. Quelque, qui avait le sens négatif, à force d'entrer dans des phrases positives, a pris le sens positif ; aucun qui avait le sens positif, à force d'entrer dans des phrases négatives, a pris le sens négatif ». (Foulet, $1919: 230$ )

«C'est par suite de son emploi fréquent dans les phrases négatives que aucun a pris le sens de «nul». C'est un fait de contagion ». (Brunot \& Bruneau, $1969: 470$ )

«Aucun conserve encore son sens primitif au XVII ${ }^{\mathrm{e}}$ siècle : [...]. Mais il commence à passer du sens positif au sens négatif en français classique, par suite de son fréquent emploi dans les phrases négatives ». (Ménard, 1973 : 39)

«Mais étant le plus souvent accompagné de la négation, aucun a pris, par contagion, la valeur négative de « pas un » ». (Grevisse, $1990: 131$ )

«Comme d'autres auxiliaires de la négation, aucun a pris par contagion le sens négatif de l'adverbe ne qu'il accompagne d'ordinaire ». (Grevisse, 1993 : 931)

Brunot \& Bruneau (1969: 472), remarquant l'effet puissant de la négation, vont jusqu'à donner la règle suivante :

«La plupart des mots-outils qui désignent, en français moderne, les notions zéro homme, zéro chose, sont de création française. Leur histoire montre la puissance d'irradiation de la négation: tout mot-outil qui se trouve habituellement en rapport étroit avec la négation prend une valeur négative ».

\subsection{Aucun comme premier introducteur de forclusion avec pleine valeur négative}

A côté de ces emplois avec ne marquant la valeur négative de aucun, la quantification nulle peut être opérée également, comme mentionné par Fournier (1998: 235), uniquement par aucun. C'est le cas, par exemple, des constructions elliptiques (interrogations, réponses...) que nous avons repérées à partir du XVII ${ }^{\mathrm{e}}$ siècle :

(17) Quels puissants motifs cet heureux avait-il pour risquer tout ce bonheur? aucun. (Martin du Gard, Jean Barois, 1913)

(18) Et immédiatement, il détourna la tête comme si sa voix avait fait plus de bruit, avait sonné plus fort qu'il ne l'aurait voulu. aucun bruit dans la maison. La porte de la cuisine s'était refermée, sombre à nouveau. (Coindreau, Lumière d'août, 1935) 
- Nos autorités ne vous ont rien expliqué ? aucun motif? (Schreiber, Un silence d'environ une demi-heure, 1996)

(20) Les avions minuscules, telles des mouches, tout là-haut, bombardaientils ? aucun bruit, sauf le grouillement du chantier. (Schreiber, Un silence d'environ une demi-heure, 1996)

Pour résumer, comme nous l'avons décrit plus haut et comme le montrent Martineau \& Déprez (2004 : 41), le français classique constitue « un point tournant » quant à l'évolution sémantique de l'indéfini aucun: diminution graduelle au cours du XVIII siècle de aucun comme terme de polarité «au profit d'un emploi comme terme négatif, probablement à cause de la cooccurrence de plus en plus fréquente de [ce terme] avec la particule ne» :

\begin{abstract}
«On [a vu] par l'étymologie et par l'historique, que aucun a essentiellement un sens affirmatif ; que le sens négatif ne lui vient que par son adjonction avec la négation ne; et que, si la fréquence de cette adjonction a altéré la netteté de la signification primitive, elle ne l'a pas détruite en fait, et surtout ne doit pas la faire perdre de vue ». (Littré, $1873: 240$ )
\end{abstract}

La valeur positive de aucun n'est plus possible aujourd'hui que dans des contextes forclusifs (contextes de polarité) : des propositions interrogatives directes/indirectes ; une subordonnée régie par une principale négative; la présence d'adjectif, verbe ou nom " forcluant»; la préposition ou la locution «forcluante » sans (que); les locutions avant (de, que), loin (de, que) ; la locution adverbiale comme si ; des constructions comparatives et des constructions hypothétiques. On retrouve ces différents contextes en français moderne mais à moindre fréquence avec une nette supériorité des emplois avec sans.

\title{
3 Aucun, évolution catégorielle
}

Outre son évolution sémantique, aucun présente cette capacité d'être polycatégoriel ${ }^{13}$ : il peut s'employer soit comme déterminant indéfini (aucun inconvénient), soit comme pronom indéfini (aucun de ces coups). Le tableau (1) et le graphe (1) permettent d'avoir une vue d'ensemble de l'évolution diachronique de ces deux emplois :

Tableau 1. Aucun, emplois déterminatifs/pronominaux.

\begin{tabular}{|c|c|c|c|}
\hline Siècle & Déterminant & Pronom & Total \\
\hline XII $^{\mathrm{e}}$ & 86 & 30 & 116 \\
\hline $\mathrm{XIII}^{\mathrm{e}}$ & 773 & 376 & 1149 \\
\hline $\mathrm{XIV}^{\mathrm{e}}$ & 4006 & 1649 & 5655 \\
\hline $\mathrm{XV}^{\mathrm{e}}$ & 3073 & 1541 & 4614 \\
\hline $\mathrm{XVI}^{\mathrm{e}}$ & 3050 & 1345 & 4395 \\
\hline $\mathrm{XVII}^{\mathrm{e}}$ & 9569 & 1927 & 11496 \\
\hline $\mathrm{XVIII}^{\mathrm{e}}$ & 14841 & 3639 & 18480 \\
\hline $\mathrm{XIX}^{\mathrm{e}}$ & 24831 & 4760 & 29591 \\
\hline $\mathrm{XX}^{\mathrm{e}}$ & 35628 & 5953 & 41581 \\
\hline
\end{tabular}

Nous constatons que les emplois pronominaux représentent le tiers des emplois déterminatifs jusqu'au XVI ${ }^{\mathrm{e}}$ siècle $\left(30,35 \%\right.$ contre $69,65 \%$ entre le $\mathrm{XII}^{\mathrm{e}}$ et le XVI ${ }^{\mathrm{e}}$ siècle). A partir du XVII ${ }^{\mathrm{e}}$ siècle, l'écart entre les deux emplois a nettement augmenté : une nette 
supériorité de aucun utilisé comme déterminant (84,8\% contre 15,2\% en français moderne) :

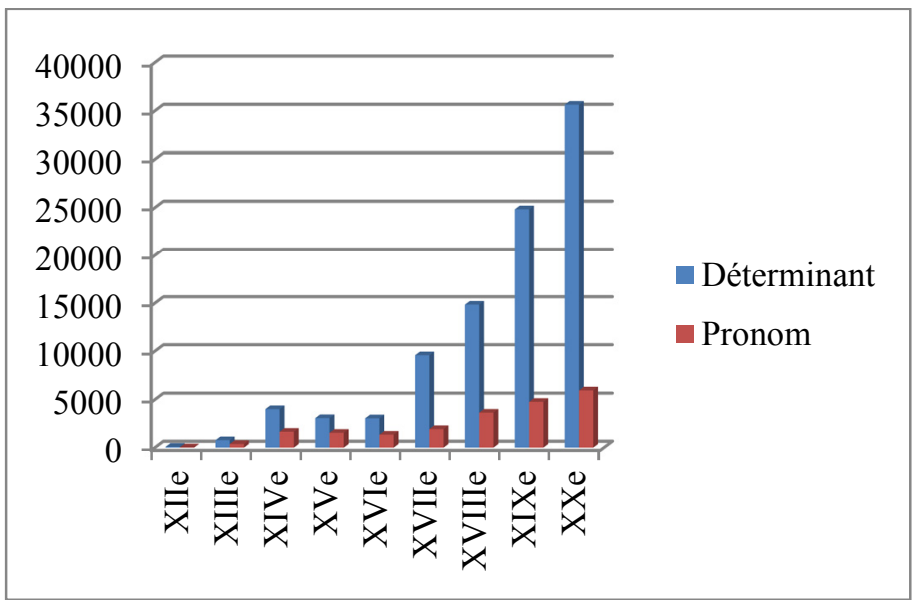

Figure 1. Aucun, évolution diachronique des emplois déterminatifs et pronominaux.

\section{Aucun, évolution morphologique}

En plus de son évolution catégorielle en tant que déterminant ou pronom, aucun présente la possibilité, du côté de son évolution morphologique, de varier en genre (masculin/féminin) et en nombre (singulier/pluriel).

\subsection{Variation en genre}

Aucun, comme d'autres indéfinis en -un en l'occurrence chacun, se caractérise par sa variation en genre (aucun ennui, aucune lutte). Le tableau (2) et le graphe (2) permettent de suivre son évolution diachronique :

Tableau 2. Aucun, variation en genre.

\begin{tabular}{|c|c|c|c|}
\hline Siècle & Masculin & Féminin & Total \\
\hline $\mathrm{XII}^{\mathrm{e}}$ & 76 & 40 & 116 \\
\hline $\mathrm{XIII}^{\mathrm{e}}$ & 689 & 460 & 1149 \\
\hline $\mathrm{XIV}^{\mathrm{e}}$ & 2979 & 2676 & 5655 \\
\hline $\mathrm{XV}^{\mathrm{e}}$ & 2816 & 1798 & 4614 \\
\hline $\mathrm{XVI}^{\mathrm{e}}$ & 2630 & 1765 & 4395 \\
\hline $\mathrm{XVII}^{\mathrm{e}}$ & 6182 & 5314 & 11496 \\
\hline $\mathrm{XVIII}^{\mathrm{e}}$ & 10090 & 8390 & 18480 \\
\hline $\mathrm{XIX}^{\mathrm{e}}$ & 15366 & 14225 & 29591 \\
\hline $\mathrm{XX}^{\mathrm{e}}$ & 21761 & 19820 & 41581 \\
\hline
\end{tabular}

Les emplois de aucun au masculin (aucun) et au féminin (aucune) coexistent en allant d'un siècle à un autre avec une légère supériorité des emplois au masculin : 


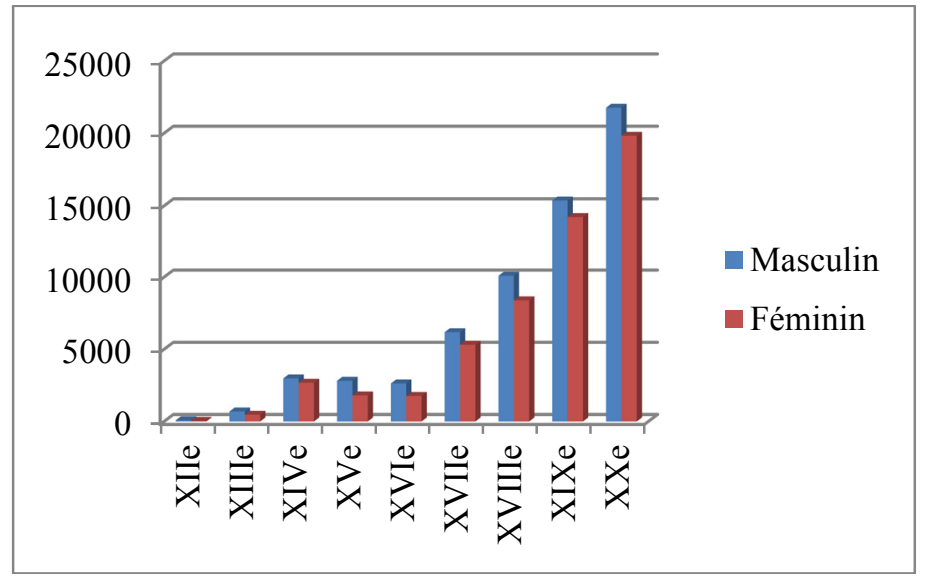

Figure 1. Aucun, évolution diachronique en genre.

\subsection{Variation en nombre : aucuns, les aucuns, d'aucuns}

Outre sa variation en genre, aucun se caractérise par sa variation en nombre ${ }^{14}$ (aucun, aucuns). Le tableau (3) et le graphe (3) permettent de tracer l'évolution diachronique des emplois de aucun au singulier par rapport à ses emplois au pluriel :

Tableau 3. Aucun, variation en nombre.

\begin{tabular}{|c|c|c|c|}
\hline Siècle & Singulier & Pluriel & Total \\
\hline $\mathrm{XII}^{\mathrm{e}}$ & 112 & 4 & 116 \\
\hline $\mathrm{XIII}^{\mathrm{e}}$ & 989 & 160 & 1149 \\
\hline $\mathrm{XIV}^{\mathrm{e}}$ & 3474 & 2181 & 5655 \\
\hline $\mathrm{XV}^{\mathrm{e}}$ & 1963 & 2651 & 4614 \\
\hline $\mathrm{XVI}^{\mathrm{e}}$ & 2820 & 1575 & 4395 \\
\hline $\mathrm{XVII}^{\mathrm{e}}$ & 10177 & 1319 & 11496 \\
\hline $\mathrm{XVIII}^{\mathrm{e}}$ & 18025 & 455 & 18480 \\
\hline $\mathrm{XIX}^{\mathrm{e}}$ & 29310 & 281 & 29591 \\
\hline $\mathrm{XX}^{\mathrm{e}}$ & 41233 & 348 & 41581 \\
\hline
\end{tabular}

Notons que c'est uniquement au $\mathrm{XV}^{\mathrm{e}}$ siècle que les emplois au pluriel s'avèrent légèrement supérieurs aux emplois au singulier. Par ailleurs, nous constatons une nette supériorité des emplois au singulier au cours des siècles suivants notamment au XVII ${ }^{\mathrm{e}}$ siècle, ce qui est expliqué par l'évolution sémantique de aucun qui bascule, comme nous l'avons vu dans le point 2 , à ce moment-là : 


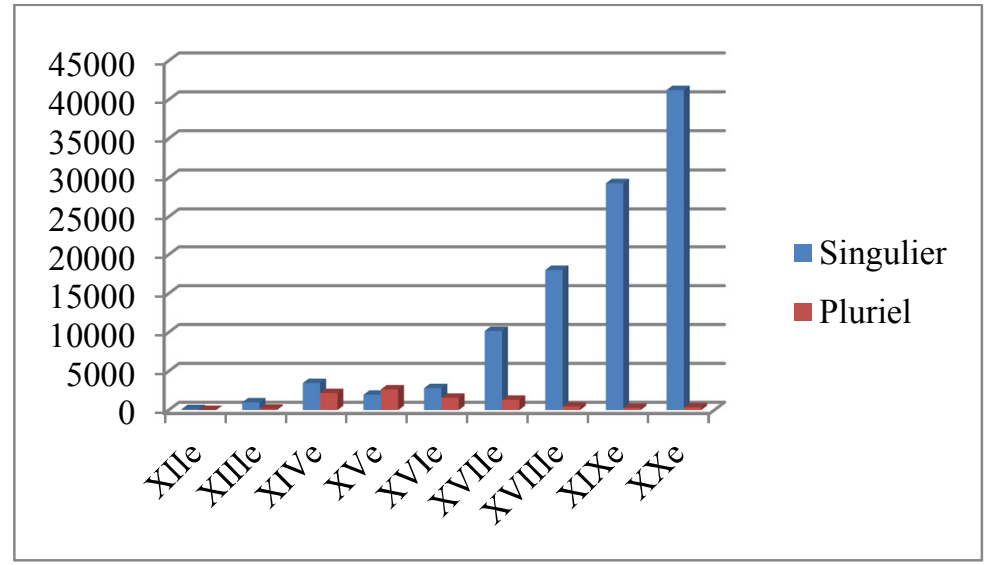

Figure 3. Aucun, évolution diachronique des emplois au singulier et au pluriel.

En consultant notre corpus et en se référant à l'étude réalisée par Prévost \& Schnedecker (2004) sur l'évolution diachronique de aucun, nous constatons que le pluriel peut se manifester, de l'ancien français au français moderne, sous plusieurs formes à savoir aucuns, les aucuns et d'aucuns. Le tableau (4) permet de tracer l'évolution de chacune de ces formes tout en précisant leurs emplois en tant que déterminant (D) et pronom $(\mathrm{P})$ :

Tableau 4. Aucun, évolution diachronique des formes plurielles.

\begin{tabular}{|c|c|c|c|c|c|c|c|c|c|}
\hline \multirow{2}{*}{ Siècle } & \multicolumn{3}{|c|}{ Les aucuns } & \multicolumn{3}{c|}{ D'aucuns } & \multicolumn{4}{c|}{ Aucuns } \\
\cline { 2 - 10 } & $\mathrm{D}$ & $\mathrm{P}$ & Total & $\mathrm{D}$ & $\mathrm{P}$ & Total & $\mathrm{D}$ & $\mathrm{P}$ & Total \\
\hline $\mathrm{XII}^{\mathrm{e}}$ & 0 & 0 & 0 & 0 & 0 & 0 & 3 & 1 & 4 \\
\hline $\mathrm{XIII}^{\mathrm{e}}$ & 0 & 23 & 23 & 1 & 0 & 1 & 100 & 36 & 136 \\
\hline $\mathrm{XIV}^{\mathrm{e}}$ & 14 & 136 & 150 & 3 & 2 & 5 & 1154 & 872 & 2026 \\
\hline $\mathrm{XV}^{\mathrm{e}}$ & 4 & 114 & 118 & 0 & 12 & 12 & 1340 & 1181 & 2521 \\
\hline $\mathrm{XVI}^{\mathrm{e}}$ & 0 & 39 & 39 & 30 & 57 & 87 & 617 & 832 & 1449 \\
\hline $\mathrm{XVII}^{\mathrm{e}}$ & 0 & 22 & 22 & 10 & 43 & 53 & 638 & 606 & 1244 \\
\hline $\mathrm{XVIII}^{\mathrm{e}}$ & 0 & 0 & 0 & 0 & 0 & 0 & 351 & 104 & 455 \\
\hline $\mathrm{XIX}^{\mathrm{e}}$ & 0 & 0 & 0 & 0 & 46 & 46 & 188 & 47 & 235 \\
\hline $\mathrm{XX}^{\mathrm{e}}$ & 0 & 0 & 0 & 1 & 279 & 280 & 60 & 8 & 68 \\
\hline Total $^{2}$ & 18 & 334 & 352 & 45 & 439 & 484 & 4451 & 3687 & 8138 \\
\hline
\end{tabular}

Les aucuns a connu des proportions relativement importantes au cours des $\mathrm{XIV}^{\mathrm{e}}$ et $\mathrm{XV}^{\mathrm{e}}$ siècles pour régresser ensuite aux $\mathrm{XVI}^{\mathrm{e}}$ et $\mathrm{XVII}^{\mathrm{e}}$ siècles et finir par disparaître au $\mathrm{XVIII}^{\mathrm{e}}$ siècle. Les emplois en tant que déterminant sont minoritaires (18 attestations) par rapport aux emplois pronominaux (334 attestations).

La forme d'aucuns, quant à elle, a suivi une évolution progressive pour atteindre son maximum au cours du $\mathrm{XX}^{\mathrm{e}}$ siècle. D'aucuns se caractérise par une nette supériorité de ses emplois pronominaux (439 occurrences) par rapport à ses emplois déterminatifs (45 occurrences). En tant que déterminant, d'aucuns a connu un pic au XVI ${ }^{\mathrm{e}}$ siècle suite auquel la forme commence sa régression jusqu'à finir par disparaître mise à part une seule attestation repérée en français moderne considérée comme archaïque. Les emplois de d'aucuns, en tant que pronom, sont plus abondants surtout au $\mathrm{XX}^{\mathrm{e}}$ siècle. 
Aucuns, finalement, ayant le plus grand nombre d'occurrences, se caractérise par sa régression au fil du temps pour aboutir à des attestations minoritaires au cours du $\mathrm{XX}^{\mathrm{e}}$ siècle. Nous constatons une légère supériorité des emplois déterminatifs (4451 occurrences) par rapport aux emplois pronominaux (3687 occurrences). Les deux emplois progressent quantitativement en allant du XII ${ }^{\mathrm{e}}$ siècle jusqu'au $\mathrm{XV}^{\mathrm{e}}$ siècle pour commencer à régresser progressivement jusqu'à devenir minoritaires au cours du $\mathrm{XX}^{\mathrm{e}}$ siècle avec une supériorité des emplois déterminatifs ( 60 occurrences) sur les emplois pronominaux ( 8 occurrences).

Pour résumer, l'étude des caractéristiques morphologiques de aucun nous a permis d'aboutir aux deux conclusions suivantes : coexistence jusqu'au français moderne des deux formes aucun et aucune et réduction des formes aucun au pluriel. En effet, les trois formes $\mathrm{du}$ pluriel aucun(e)s, d'aucun(e)s et les aucuns ont subi plusieurs changements. Nous aboutissons à la disparition de les aucuns au XVII ${ }^{\mathrm{e}}$ siècle et au maintien des deux autres formes (aucuns et d'aucuns) avec, en revanche, une réduction de leurs caractéristiques. Hormis la persistance de certaines attestations archaïques, vieillies, relevant du style marotique, nous notons la disparition de aucuns en emploi pronominal et d'aucuns en emploi déterminatif. D'aucuns, en emploi pronominal, acquiert, quant à lui, un double avis contradictoire dans la mesure où certains grammairiens le considèrent comme vieilli ${ }^{15}$, là où d'autres notent qu'il est tout à fait en usage ${ }^{16}$. On pourrait expliquer la régression des emplois au pluriel de aucun par la disparition de sa valeur positive. Suite à la réanalyse de aucun comme indéfini négatif, les grammairiens se sont mis à rejeter l'emploi pluriel parce qu'il n'est pas logique : aucun signifiant alors "pas un »; s'il n'y en a pas un, on ne voit pas pourquoi on devrait utiliser le pluriel. Wagner \& Pinchon (1962) notent que le pluriel aucun(e)s ne s'est conservé que dans les phrases positives. Notre corpus nous montre, néanmoins, que cette forme continue à s'employer en français moderne non seulement dans des phrases positives mais également dans des phrases négatives :

(21) Aujourd'hui je me borne à me réjouir sincèrement qu'aucuns de ces personnages ne s'interposent plus entre Mansart, Robert De Cotte, Mique, Lenôtre, Tuby, Coisevox et ma solitude. (du Bos, Journal : t. 4, 1928)

Littré (1873 : 240) va jusqu'à légitimer ces emplois négatifs au pluriel :

"Quelques personnes doutent si aucun, aucune, avec la négation, peuvent être employés au pluriel. Il est plus ordinaire de mettre le singulier ; mais comme rien n'empêche de nier la pluralité aussi bien qu'on nie l'unité, rien non plus ne peut faire condamner les phrases où aucun est au pluriel. On voit par les exemples que les meilleurs auteurs, en prose comme en vers, se sont servis d'aucun au pluriel. Cet emploi est donc pleinement légitime ».

\section{Aucun, évolution syntaxique}

En dehors de son évolution morphologique (variation en genre et en nombre), aucun a suivi une évolution syntaxique se résumant, entre autres, dans les trois points suivants : les cas de postposition $(\mathrm{N}+$ aucun), les cas d'insertion (aucun + adjectif/indéfini $+\mathrm{N}$ ) et les constructions directes/indirectes des adjectifs/indéfinis joints à aucun (aucun + adjectif/indéfini vs aucun + de + adjectif/indéfini).

\subsection{Cas de postposition : $\mathrm{N}+$ aucun}

Dans l'usage actuel, l'indéfini aucun est généralement antéposé au nom. En témoigne, comme le montrent le tableau (5) et le graphe (4), la nette supériorité des cas d'antéposition par rapport aux cas de postposition : 
Tableau 5. Evolution diachronique de la position de aucun.

\begin{tabular}{|c|c|c|}
\hline Siècle & aucun Nom & Nom aucun \\
\hline $\mathrm{XII}^{\mathrm{e}}$ & 116 & 0 \\
\hline $\mathrm{XIII}^{\mathrm{e}}$ & 1147 & 2 \\
\hline $\mathrm{XIV}^{\mathrm{e}}$ & 5547 & 108 \\
\hline $\mathrm{XV}^{\mathrm{e}}$ & 4549 & 65 \\
\hline $\mathrm{XVI}^{\mathrm{e}}$ & 4185 & 210 \\
\hline $\mathrm{XVII}^{\mathrm{e}}$ & 11351 & 145 \\
\hline $\mathrm{XVIII}^{\mathrm{e}}$ & 18453 & 27 \\
\hline $\mathrm{XIX}^{\mathrm{e}}$ & 29360 & 231 \\
\hline $\mathrm{XX}^{\mathrm{e}}$ & 41178 & 403 \\
\hline
\end{tabular}

Nous constatons, au fil du temps, une nette supériorité des cas d'antéposition par rapport aux cas de postposition, témoignant ainsi d'une tendance vers la fixation de la position de aucun malgré les 634 cas de postposition relevés aux $\mathrm{XIX}^{\mathrm{e}}$ et $\mathrm{XX}^{\mathrm{e}}$ siècles, un nombre qui a augmenté légèrement par rapport aux siècles précédents, mais qui demeure minime.

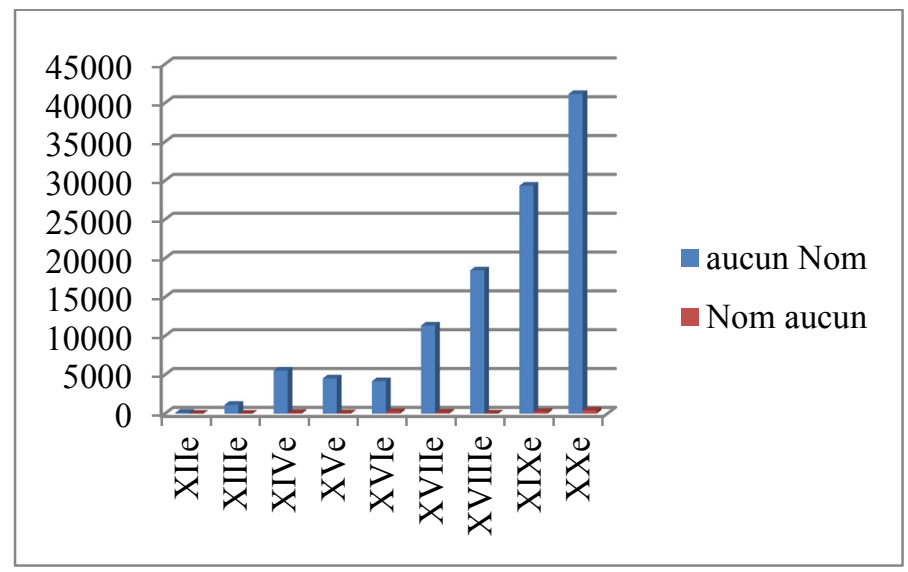

Figure 4. Aucun $\mathrm{N}$ et $\mathrm{N}$ aucun : évolution diachronique.

Aucun peut être postposé, selon Chevalier et al. (1964: 266) ou Grevisse (1993: 31), lorsque le substantif est précédé de la préposition sans. En dehors de cette construction, d'autres cas de postposition peuvent avoir lieu, ils sont considérés alors, selon les termes de Grevisse (1993 : 932), comme « en marge de l'usage ».

L'exploration de notre corpus nous a permis de constater que la postposition concerne effectivement ces deux cas (sans rapport aucun; rapport aucun) que nous résumons dans le tableau (6) :

Tableau 6. Evolution diachronique des cas de postposition de aucun.

\begin{tabular}{|c|c|c|c|}
\hline Siècle & Sans $+\mathrm{N}+$ aucun & $\mathrm{N}+$ aucun & Total \\
\hline $\mathrm{XIII}^{\mathrm{e}}$ & 0 & 2 & 2 \\
\hline $\mathrm{XIV}^{\mathrm{e}}$ & 41 & 67 & 108 \\
\hline
\end{tabular}




\begin{tabular}{|c|c|c|c|}
\hline $\mathrm{XV}^{\mathrm{e}}$ & 22 & 43 & 65 \\
\hline $\mathrm{XVI}^{\mathrm{e}}$ & 54 & 156 & 210 \\
\hline $\mathrm{XVII}^{\mathrm{e}}$ & 75 & 70 & 145 \\
\hline $\mathrm{XVIII}^{\mathrm{e}}$ & 9 & 18 & 27 \\
\hline $\mathrm{XIX}^{\mathrm{e}}$ & 224 & 7 & 231 \\
\hline $\mathrm{XX}^{\mathrm{e}}$ & 388 & 15 & 403 \\
\hline
\end{tabular}

Les cas de postposition ont suivi, comme le montre le graphe (5), l'évolution suivante : les deux cas progressent jusqu'aux $\mathrm{XVI}^{\mathrm{e}}(\mathrm{N}+$ aucun $)$ et $\mathrm{XVII}^{\mathrm{e}}($ sans $+\mathrm{N}+$ aucun $)$ siècles ; nous observons ensuite une nette chute de la construction (sans $+\mathrm{N}+$ aucun) au XVIII ${ }^{\mathrm{e}}$ siècle et une régression de la construction $(\mathrm{N}+$ aucun $)$ à partir du XVII ${ }^{\mathrm{e}}$ siècle; nous constatons enfin que les constructions précédées de sans reprennent leur progression alors que les autres cas se marginalisent :

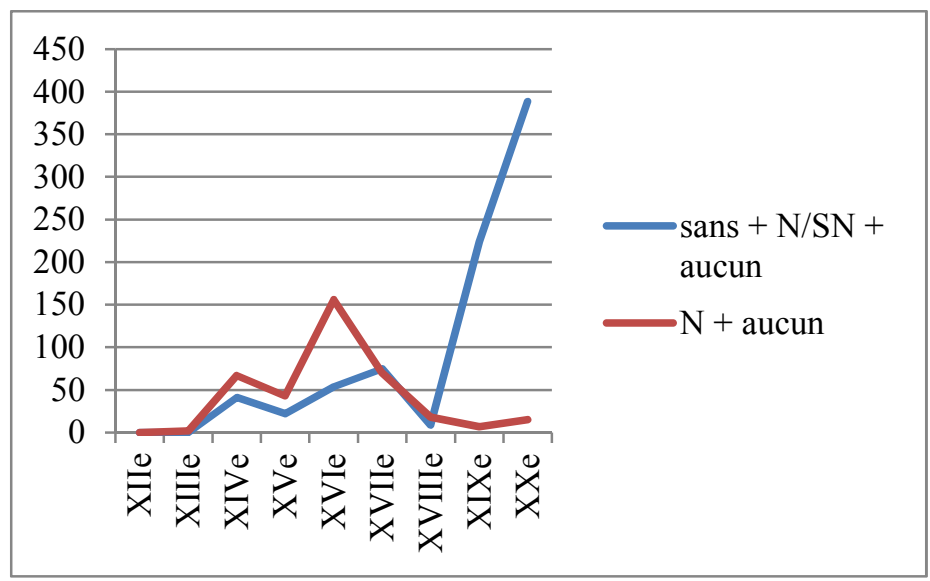

Figure 5. Evolution des cas de postposition de aucun.

Les constructions $\mathrm{N}+$ aucun se trouvent $\mathrm{du} \mathrm{XIV}^{\mathrm{e}}$ siècle jusqu'au XVII ${ }^{\mathrm{e}}$ siècle essentiellement dans des phrases où règne une atmosphère négative : 48 attestations de valeur négative sur un total de 67 occurrences au XIV ${ }^{\mathrm{e}}$ siècle ; 32 sur un total de 43 au XV siècle ; 134 sur un total de 156 au XVI ${ }^{\mathrm{e}}$ siècle et 69 sur un total de 70 au XVII ${ }^{\mathrm{e}}$ siècle. Cette valeur négative est visible dans des phrases introduites par la particule de négation ne. Au cours des siècles suivants, nous n'avons relevé que des attestations négatives introduites par ne:

(22) [...] voilà que je n'ai plus de rapport aucun à la notion de prix. Adieu l'argent. (Pachet, Autobiographie de mon père, 1987)

Concernant les autres cas de postposition sans $+\mathrm{N}+$ aucun, Grevisse (1993: 31) note que aucun n'est plus considéré comme un déterminant, mais plutôt comme un adjectif épithète : « quand le syntagme est précédé de sans (dont le régime se construit souvent sans déterminant) ; il cesse alors d'être un déterminant $»$ :

(23) Anna n'avait d'italien que le minois, trop naturelle dans ses humeurs comme dans ses gestes, sans coquetterie aucune, avec un enthousiasme tempéré par la tendresse. (Rambaud, La Bataille, 1997)

Ceci contredit ce qu'avancent, quelques années plus tôt, Grevisse \& Goosse (1989: 185) concernant ce changement de valeur : «[a]ucun, au contraire des autres déterminants, peut se placer après le nom, dans la langue écrite, sans changer de valeur. Cela se produit 
uniquement quand le syntagme est introduit par la préposition sans». Ces deux avis partagés entre aucun comme déterminant ou comme adjectif épithète montrent qu'il y a un problème concernant le statut catégoriel de aucun.

\subsection{Cas d'insertion : aucun + adjectif/indéfini $+\mathbf{N}$}

En plus de ces cas de postposition $(\mathrm{N}+$ aucun), le groupe nominal aucun $\mathrm{N}$ se caractérise par la possibilité d'insertion d'adjectifs ou d'indéfinis entre ses deux constituants. Le tableau (7) et le graphe (6) permettent de suivre leur évolution diachronique :

Tableau 7. Aucun, évolution diachronique des cas d'insertion.

\begin{tabular}{|c|c|c|}
\hline Siècle & Aucun + adjectif/indéfini $+\mathrm{N}$ & Total \\
\hline $\mathrm{XII}^{\mathrm{e}}$ & 4 & 116 \\
\hline $\mathrm{XIII}^{\mathrm{e}}$ & 69 & 1149 \\
\hline $\mathrm{XIV}^{\mathrm{e}}$ & 280 & 5655 \\
\hline $\mathrm{XV}^{\mathrm{e}}$ & 210 & 4614 \\
\hline $\mathrm{XVI}^{\mathrm{e}}$ & 141 & 4395 \\
\hline $\mathrm{XVII}^{\mathrm{e}}$ & 477 & 11496 \\
\hline $\mathrm{XVIII}^{\mathrm{e}}$ & 565 & 18480 \\
\hline $\mathrm{XIX}^{\mathrm{e}}$ & 813 & 29591 \\
\hline $\mathrm{XX}^{\mathrm{e}}$ & 945 & 41581 \\
\hline
\end{tabular}

Ces différents cas d'insertion ne cessent généralement d'augmenter au fil des siècles :

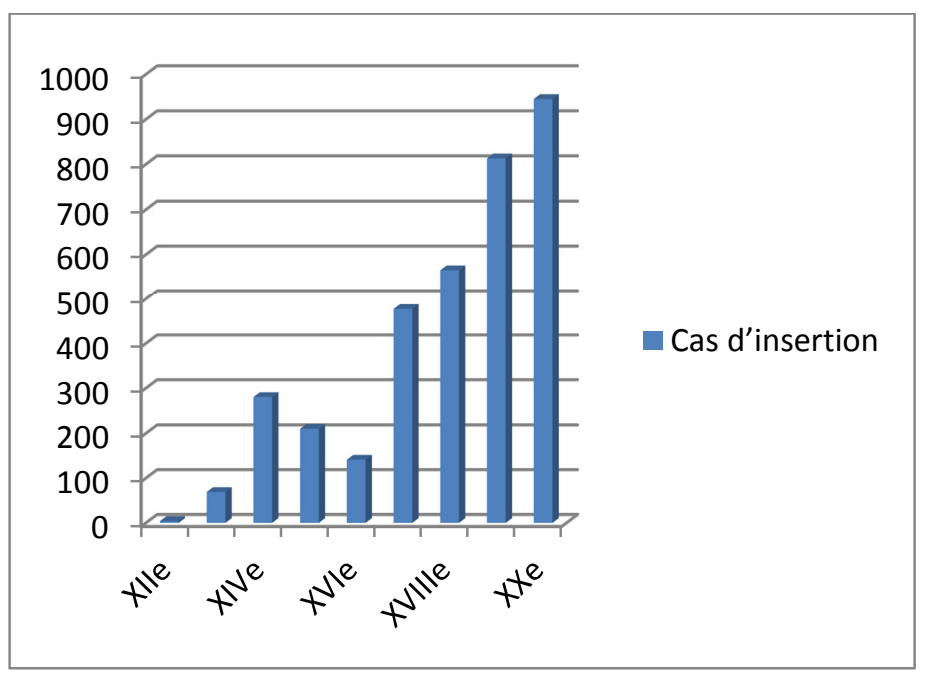

Figure 6. Aucun, évolution diachronique des cas d'insertion.

$\mathrm{Au}$ cours du XII ${ }^{\mathrm{e}}$ siècle, nous n'avons relevé que quatre cas d'insertion avec les adjectifs bon et grand. Au cours des siècles suivants, nous constatons une augmentation progressive en nombre d'occurrences comme le montre le tableau (7). Notons également une diversité au niveau des mots inséré ${ }^{17}$ avec des proportions variables au fil des siècles. $\mathrm{Au}$ cours de chaque siècle, nous avons une reproduction du schéma suivant que nous résumons dans le tableau (8) : dominance de l'insertion de l'indéfini autre suivi des 
adjectifs bon, grand, jeune, mauvais, nouveau, véritable et vrai (leur nombre d'occurrences varie selon les synchronies) et insertion d'autres adjectifs ${ }^{18}$ représentant des cas minoritaires avec une utilisation limitée réduite généralement à un ou deux cas :

Tableau 8. Evolution diachronique des adjectifs/indéfinis insérés.

\begin{tabular}{|c|c|c|c|c|c|c|c|c|c|}
\hline \multirow{2}{*}{ Siècle } & \multirow{2}{*}{ autre } & \multicolumn{7}{|c|}{ Les adjectifs majoritaires } & \multirow{2}{*}{$\begin{array}{c}\text { Les cas } \\
\text { minoritaires }\end{array}$} \\
\cline { 3 - 10 } & & bon & grand & jeune & mauvais & nouveau & véritable & vrai & \\
\hline $\mathrm{XIII}^{\mathrm{e}}$ & 22 & 6 & 11 & 0 & 2 & 1 & 0 & 0 & 27 \\
\hline $\mathrm{XIV}^{\mathrm{e}}$ & 127 & 21 & 24 & 5 & 5 & 4 & 0 & 0 & 94 \\
\hline $\mathrm{XV}^{\mathrm{e}}$ & 44 & 47 & 27 & 6 & 7 & 8 & 0 & 1 & 70 \\
\hline $\mathrm{XVI}^{\mathrm{e}}$ & 33 & 19 & 9 & 3 & 12 & 4 & 0 & 3 & 58 \\
\hline $\mathrm{XVII}^{\mathrm{e}}$ & 248 & 37 & 11 & 2 & 47 & 13 & 6 & 15 & 98 \\
\hline $\mathrm{XVIII}^{\mathrm{e}}$ & 393 & 20 & 10 & 12 & 45 & 11 & 18 & 14 & 42 \\
\hline $\mathrm{XIX}^{\mathrm{e}}$ & 600 & 26 & 46 & 14 & 40 & 12 & 9 & 6 & 60 \\
\hline $\mathrm{XX}^{\mathrm{e}}$ & 706 & 23 & 46 & 18 & 25 & 25 & 18 & 15 & 69 \\
\hline
\end{tabular}

\subsection{Constructions directes/indirectes : aucun + adjectif/indéfini vs aucun + de + adjectif/indéfini}

A côté de ces différents cas d'insertion (aucun + adjectif/indéfini $+\mathrm{N}$ ), marqués par la diversité et la progression quantitative des mots insérés, aucun se caractérise également par la possibilité de se construire directement ${ }^{19}$ ou indirectement ${ }^{20}$ avec des adjectifs ou des indéfinis (aucun + adjectif/indéfini vs aucun $+d e+$ adjectif/indéfini). Wagner \& Pinchon (1962 : 202) notent que les deux constructions sont possibles: aucun se construit indirectement via la préposition de ou directement avec des adjectifs. Ils précisent (Ibid.) que cette "différence de construction n'entraîne aucune différence de sens. Le choix doit donc être réglé par l'oreille et d'après des convenances de style. En parlant, on utilisera de préférence la construction indirecte qui épargne des liaisons insolites ». Riegel et al. (1994: 211) remarquent, en revanche, que aucun ne peut pas se construire indirectement avec des adjectifs épithètes. Nous rejoignons l'avis de Wagner \& Pinchon puisque l'exploration de notre corpus nous a permis de constater que les deux constructions (directes et indirectes ${ }^{21}$ ) sont tout à fait possibles comme en témoignent ces différentes attestations :

(24) Il me semble que l'âme bien seule avec elle-même, et qui se parle, de temps à autre, entre deux silences absolus, n'emploie jamais qu'un petit nombre de mots, et aucun d'extraordinaire. (Valéry, Variété V, 1944)

(25) Aller s'enterrer dans une gargote de banlieue ne lui chantait guère. Des projets plus brillants, il n'en avait aucun de précis ; il était certain qu'ils s'offriraient d'eux-mêmes comme cet argent s'offrait à lui. (Bazin, La Tête contre les murs, 1949)

(26) [...] la Voie royale (financièrement la plus assurée (quoique modestement) ainsi que la plus courte (quoique relativement)) paraissait claire (d'ailleurs je n'en connaissais aucune autre) : (Roubaud, Poésie : récit, 2000)

Le tableau (9) et le graphe (7) permettent de tracer l'évolution progressive de ces différents cas utilisés à partir du XIII ${ }^{\mathrm{e}}$ siècle : 
Tableau 9. Aucun, évolution diachronique des constructions directes/indirectes avec des adjectifs/indéfinis.

\begin{tabular}{|c|c|c|c|c|}
\hline Siècle & $\begin{array}{c}\text { Aucun }+ \\
\text { adjectif/indéfini }\end{array}$ & $\begin{array}{c}\text { Aucun }+ \text { de }+ \\
\text { adjectif/indéfini }\end{array}$ & $\begin{array}{c}\text { Total des } \\
\text { constructions } \\
\text { directes et } \\
\text { indirectes }\end{array}$ & $\begin{array}{c}\text { Total de } \\
\text { aucun } \\
\text { pronom }\end{array}$ \\
\hline $\mathrm{XII}^{\mathrm{e}}$ & 0 & 0 & 0 & 30 \\
\hline $\mathrm{XIII}^{\mathrm{e}}$ & 5 & 0 & 5 & 376 \\
\hline $\mathrm{XIV}^{\mathrm{e}}$ & 71 & 0 & 71 & 1649 \\
\hline $\mathrm{XV}^{\mathrm{e}}$ & 48 & 0 & 48 & 1541 \\
\hline $\mathrm{XVI}^{\mathrm{e}}$ & 49 & 1 & 50 & 1345 \\
\hline $\mathrm{XVII}^{\mathrm{e}}$ & 207 & 8 & 215 & 1927 \\
\hline $\mathrm{XVIII}^{\mathrm{e}}$ & 242 & 24 & 266 & 3639 \\
\hline $\mathrm{XIX}^{\mathrm{e}}$ & 333 & 11 & 344 & 4760 \\
\hline $\mathrm{XX}^{\mathrm{e}}$ & 465 & 6 & 471 & 5953 \\
\hline
\end{tabular}

Nous constatons, à partir du $\mathrm{XVII}^{\mathrm{e}}$ siècle, une nette supériorité des constructions directes. L'écart entre les deux types de constructions devient de plus en plus important au fil des siècles :

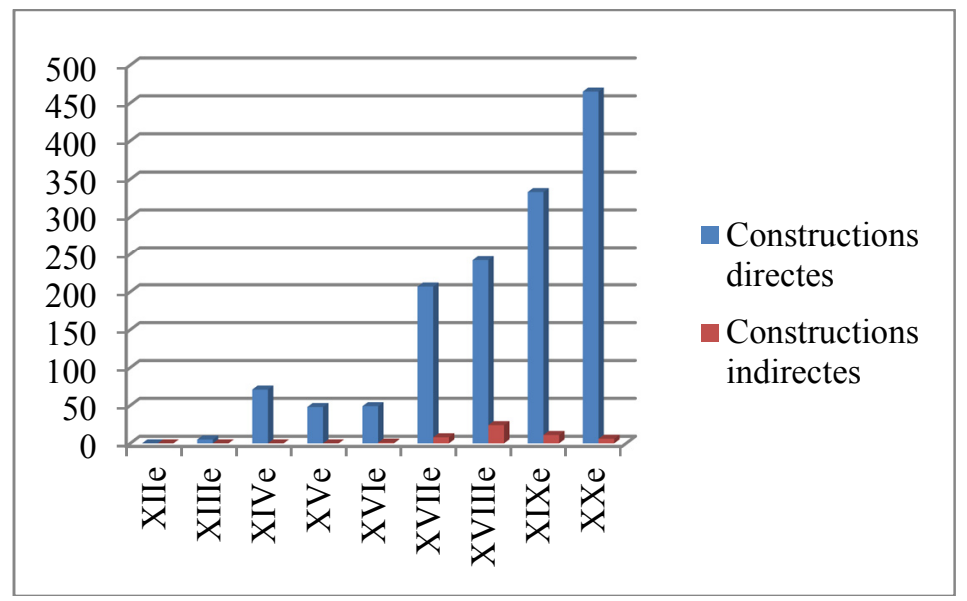

Figure 7. Aucun, évolution des constructions directes/indirectes

\section{Conclusion}

Au terme de cette étude, nous avons tenté de décrire la distribution de l'indéfini aucun d'un point de vue diachronique : évolution sémantique (valeur positive et négative), évolution catégorielle (double statut de déterminant et de pronom), évolution morphologique (variation en genre et en nombre) et évolution syntaxique (cas d'insertion (aucun + adjectif/indéfini $+\mathrm{N}$ ), de postposition ( $\mathrm{N}$ aucun) et des constructions directes/indirectes des adjectifs/indéfinis joints à aucun).

Ce travail nous a permis de repérer plusieurs changements concernant l'évolution de aucun. D'un point de vue sémantique, ce morphème peut être considéré comme un « forclusif » présentant trois valeurs bien distinctes. Il a d'abord une valeur positive qu'il 
garde dans certaines tournures (propositions interrogatives directes et indirectes; subordonnée régie par une principale négative; présence d'adjectif, verbe ou nom «forcluant»; préposition ou locution «forcluante » sans (que); locutions avant (de, que), loin (de, que); locution adverbiale comme si ; constructions comparatives et constructions hypothétiques). En dehors de ces tournures «forclusives », aucun peut avoir généralement une valeur positive lorsqu'il est employé au pluriel à travers les formes aucuns, les aucuns et d'aucuns. Il présente ensuite une valeur négative acquise suite à son emploi fréquent avec la particule de négation ne. Il a enfin une valeur pleinement négative grâce à son emploi « nu » par exemple dans des constructions elliptiques. Avec aucun, nous assistons à une évolution d'une valeur positive assez fréquente jusqu'au français classique, comme l'observent Damourette \& Pichon (1911-1940 : § 2917) : «Un simple coup d'œil lancé sur l'ancienne langue montre que les emplois positifs d'aucun étaient assez fréquents jusqu'à l'orée de l'âge classique », vers une valeur négative.

L'évolution catégorielle de aucun nous a montré que son emploi pronominal s'est restreint. Aucun est en train de chercher une certaine stabilisation visible grâce à la nette supériorité des emplois déterminatifs. Les prochains siècles seraient les seuls témoins permettant d'infirmer ou de confirmer son emploi exclusivement comme déterminant.

Côté morphologie, nous remarquons une réduction formelle des cas au pluriel. Cette diminution importante du pluriel serait due à deux facteurs : 1) la réanalyse de aucun comme terme négatif, avec pour conséquence 2) l'illogisme du pluriel selon les grammairiens. Dans le sens positif, nous passons des trois formes aucuns, les aucuns et d'aucuns à pratiquement la forme d'aucuns ${ }^{22}$ dotée d'une valeur positive et fonctionnant actuellement comme un pronom indéfini à part entière.

Sur le plan syntaxique, nous constatons une tendance vers la fixation de la position de aucun : nette supériorité des emplois antéposés $($ aucun $+\mathrm{N})$ sur les emplois postposés $(\mathrm{N}+$ aucun). Les cas d'insertion (aucun + adjectif/indéfini $+\mathrm{N}$ ) sont marqués par la diversité et la progression quantitative des mots insérés, avec notamment la dominance de l'insertion de l'indéfini autre. Concernant la construction directe ou indirecte de aucun avec des adjectifs/indéfinis, notons que les deux cas sont possibles avec dominance des constructions directes : dans les comparatives, on privilégie les constructions directes (plus qu'aucune autre, aussi qu'aucune autre...); les constructions indirectes, quant à elles, renvoient généralement à $e n$.

\section{Références bibliographiques}

Arrivé, M., Gadet, F. \& Galmiche, M. (1986). La grammaire d'aujourd'hui : guide alphabétique de linguistique française. Paris : Flammarion.

Brunot, F. (1966). Histoire de la langue française des origines à nos jours : Le XVIII siècle. Paris : Armand Colin.

Brunot, F. \& Bruneau, Ch. (1969). Précis de grammaire historique de la langue française. Paris : Masson et $\mathrm{C}^{\mathrm{ie}}$.

Buridant, Cl. (2000). Les quantificateurs indéfinis. Grammaire nouvelle de l'ancien français. Paris : Sedes.

Chevalier, J.-C., Blanche-Benveniste, C., Arrivé, M. \& Peytard, J. (1964). Grammaire du français contemporain. Paris : Larousse.

Damourette, J. \& Pichon, E. (1911-1940). Des mots à la pensée. Essai de Grammaire de la Langue Française. Paris : D’Artrey.

Féraud, J.-F. (1787). Dictionnaire critique de la langue française. Marseille : Mossy.

Floricic, F. (2009). Pourquoi Nessun/Nessuno en italien mais *Auque/Aucun en français ?*. Studia Linguistica, 126, 33-45.

Foulet, L. (1919). Études de syntaxe française : I. Quelque. Romania, 178, 220-249.

Fournier, N. (1998). Grammaire du français classique. Paris : édition Belin.

Grevisse, M. (1990). Précis de grammaire française. Paris : Duculot.

Grevisse, M. (1993). Le Bon Usage. Paris : Duculot.

Grevisse, M. \& Goosse, A. (1989). Nouvelle Grammaire française. Paris : Duculot. 
Haspelmath, M. (1997). Indefinite Pronouns. Oxford : Clarendon Press.

Jespersen, O. (1971). La philosophie de la grammaire. Paris : Minuit.

Le Bidois, G. \& Le Bidois, R. (1967). Syntaxe du français moderne, ses fondements historiques et psychologiques. Paris : éditions Alphonse et Jacques Picard et $C^{\text {ie }}$.

Littré, E. (1873). Dictionnaire de la langue française. Paris : Hachette.

Marchello-Nizia, Ch. (1997). Adjectifs, pronoms et adverbes indéfinis. Histoire de la Langue française aux $X I V^{e}$ et $X V^{e}$ siècles. Paris : Nathan.

Martineau, F. \& Déprez, V. (2004). Pas rien/Pas aucun en français classique : variation dialectale et historique. Langue française, 143, 33-47.

Ménard, Ph. (1973). Manuel du français du moyen âge. I. Syntaxe de l'ancien français. Bordeaux : Sobodi.

Picoche, J. \& Marchello-Nizia, Ch. (2008). Histoire de la langue française. Paris : éditions VIGDOR.

Pierrard, M. (1998). Syntagme nominal et pronoms : la grammaticalisation des pronoms « essentiels » indéfinis. La ligne claire de la linguistique à la grammaire. Paris-Bruxelles : Duculot.

Prévost, S. \& Schnedecker, C. (2004) Aucun $(e)(s) / D$ 'aucun $(e)(s) /$ Les aucun $(e)(s)$ : évolution du français médiéval au français moderne. Scolia, 18, 41-75.

Riegel, M., Pellat, J.-Ch. \& Rioul, R. (1994). Grammaire méthodique du français. Paris : PUF.

Sancier-Chateau, A. (1993). Introduction à la langue du XVII siècle. Paris : Nathan.

Spillebout, G. (1985). Grammaire de la langue française du XVII siècle. Paris : Picard.

Trésor de la Langue Française informatisé (TLFi). Site internet : $<\mathrm{http}: / /$ www.atilf.fr/tlfi $>$.

Wagner, R.-L. \& Pinchon, J. (1962). Grammaire du français classique et moderne. Paris : Hachette universelle.

\footnotetext{
${ }^{1}$ Nous n'avons pris en compte qu'une partie de chacune de ces bases de données afin d'éviter le biais du doublement de certains textes.

${ }^{2} \mathrm{http}: / / \mathrm{bfm}$. ens-lsh.frl.

3 http://www.atilf.fr/dmf/.

${ }^{4}$ http://www.frantext.fr.
}

5 Selon Fournier (1998 : 233), nous devons la notion de forclusif à Damourette \& Pichon (1911-1940, Etude générale de la langue française) qui donnent ce nom à des termes comme rien, jamais, aucun, personne, plus, guère, etc. "s'appliqu[ant] aux faits que le locuteur n'envisage pas comme faisant partie de la réalité » (Tome I, § 116). Ils ont réexaminé ces différents termes dans le tome VI (§ 2241) : «L'observation de la langue française conduit rapidement à remarquer que dans un certain type de phrases, des struments d'une série spéciale, aucun, rien, jamais, guère, plus, non plus, etc. peuvent être appelés à figurer respectivement au lieu des struments indifférenciés acceptifs : un, quelque chose, un jour, beaucoup, encore, aussi, etc. Ces struments ont pour fonction de classer ce qu'ils expriment hors du champ de ce qui est aperçu comme réel ou réalisable. [...] nous avons donné à ces struments le nom de forclusifs ». Ces termes forclusifs sont employés dans des contextes non négatifs créant toutefois une «atmosphère forclusive» qui «ne procède en réalité pas de circonstances grammaticales, mais de motifs sémantiques d'ordre psychologique. On y est plongé toutes les fois qu'on gravite autour d'un fait présenté comme ne rentrant pas dans un envisagement facile de la réalité, la réalité devant s'entendre ici, comme on va le voir, sous le triple aspect de la probabilité, de la désirableté et de l'attingibilité investigatoire» (§2244). Fournier ajoute que cette atmosphère est « créée par différents indices contextuels, qui sont responsables de ce défaut de réalité : négation pleine ne ... pas, marqueurs lexicaux (préposition, adjectif, nom ou verbe), interrogation et percontation, comparaison, hypothèse, etc., tous opérateurs qu'on peut dire forcluants dans la mesure où ils créent un obstacle à la réalisation et même à l'envisagement du procès ».

${ }^{6}$ Voir Haspelmath (1997 : 34-35) qui montre la polarité négative de la série any-en anglais dans des interrogatives, des hypothétiques, des comparatives, après sans...

${ }^{7}$ Nous empruntons le terme « forcluant(e) » à Fournier $(1998: 234)$.

${ }^{8}$ Ces adjectifs sont principalement difficile, impossible, incapable, indigne et inutile, avec une nette supériorité d'impossible et incapable.

${ }^{9}$ Parmi ces verbes, nous citons s'abstenir, défendre, douter, empêcher, éviter, exclure, se garder, interdire, nier, ôter, prohiber, refuser et renoncer, avec une supériorité des verbes défendre et empêcher.

${ }^{10}$ Ces noms se limitent à défense, impossibilité, impuissance, inaptitude, incapacité, interdiction et refus, avec une fréquence d'emploi plus élevée concernant défense, impossibilité et impuissance. 


\begin{abstract}
${ }^{11}$ Citons, par exemple, l'emploi de aucun avec une valeur positive après des verbes de parole comme dire.

${ }^{12}$ Voir 4.2 Variation en nombre : aucuns, les aucuns, d'aucuns.

${ }^{13}$ La même forme aucun fonctionne aussi bien comme déterminant ou pronom contrairement par exemple, comme le montre Floricic (2009: 39), aux indéfinis chacun/chaque et quelqu'un/quelque. Ce dernier (Ibid.) note que l'indéfini chacun qui s'employait au XV siècle « tant comme pronom que comme adjectif [...] devient exclusivement pronom au XVII ${ }^{\mathrm{e}}$ siècle (cf. Fournier 1998 : 221)»; l'indéfini chaque, apparu dès le $\mathrm{XII}^{\mathrm{e}}$ siècle, "demeure assez exceptionnel au $\mathrm{XV}^{\mathrm{e}}$ siècle et ne
\end{abstract} s'impose qu'au XVI ${ }^{\mathrm{e}}$ siècle (cf. Brunot 1967 vol. 2 : 320, Marchello-Nizia 1992 : 151) ».

${ }^{14}$ Le français moderne se caractérise par l'utilisation dominante du singulier, mais sans empêcher l'emploi du pluriel, à travers les formes aucuns et d'aucuns, sous certaines conditions se résumant dans la citation suivante : «AUCUN [...] s'emploi[e] le plus souvent au singulier. Cependant [il] s'emploi[e] au pluriel quand [il détermine] des substantifs qui n'existent qu'à ce nombre (ou qui ont un singulier un sens différent, et, uniquement dans l'usage littéraire, quand la substance dont ils marquent l'absence constitue d'ordinaire plusieurs unités : On ne peut lui attribuer aucun malaise de l'âme, aucunes ombres intérieures (Valéry)» (Chevalier et al., 1964 : 266). Selon Grevisse (1990: 104), aucun peut se mettre au pluriel avec des substantifs qui ne présentent pas de formes au singulier (frais) ou qui ont un sens bien particulier ou différent au pluriel (travaux publics). Or, ce n'est pas tout à fait exact puisque le pluriel peut se rencontrer également en dehors de ces cas bien précis dans la mesure où « [1]a langue écrite, surtout littéraire, continue à utiliser le pluriel devant » (Grevisse, 1993 : 932) des substantifs tels que désirs, plans, pieds, commentaires, ustensiles, soins, chevaux, termes, nombres, saluts, serviteurs, besoins, etc. qui présentent une forme au singulier. Féraud (1787 : 203) est pour l'emploi de aucun au singulier : "Aucun c'est, pas un. Qui n'en a pas un, n'en a point du tout : qu'a-t-on donc à faire du pluriel ? ». Selon le même auteur, l'emploi au singulier est dû, "outre l'usage», au fait que cet indéfini est "accompagné d'une particule négative ». D'Olivet (1738: 116), cité par Brunot (1966: 1659), note que aucun n'a jamais de pluriel, une « règle confirmée en 1767 [...] ; cela ne serait tolérable que dans le style marotique ou au Palais, et au sens de quelques-uns ». Ce qui rejoint l'idée que la question du pluriel est posée en rapport avec la valeur positive/négative de aucun(e)s. A ce style marotique, Spillebout (1985: 89), pour justifier l'emploi pluriel de aucun, ajoute que « certains écrivains en font une élégance ».

${ }^{15}$ Certains grammairiens considèrent d'aucuns comme vieilli. Marchello-Nizia (1997 : 188) note que d'aucun $(e) s$, très fréquent aux $\mathrm{XIV}^{\mathrm{e}}$ et $\mathrm{XV}^{\mathrm{e}}$ siècles, « ne se rencontre encore que dans quelques textes de la fin du XV $\mathrm{X}^{\mathrm{e}}$ siècle ». Brunot \& Bruneau $(1969: 471)$ avancent que cette forme est « archaïque ». Sancier-Chateau (1993: 66) observe que la « forme pronominale plurielle précédée de de survit dans la première partie du XVII ${ }^{e}$ siècle, avec le sens de quelques-uns. F. Brunot note qu'après 1650 , « on ne retrouvera [d]'aucuns ainsi employé que par archaïsme ou en burlesque »». Elle note (Ibid., 31) que son utilisation dans les Contes et les Fables de La Fontaine relève d'un «style marotique ». Littré (1873 : 240) considère également d'aucun(e)s comme un cas d'archaïsme qui n'est "plus guère en usage ».

${ }^{16} \mathrm{D}$ 'autres grammairiens considèrent d'aucuns comme un mot encore utilisé. Citons, entre autres, Le Bidois, G. \& Le Bidois, R. (1967 : 218) qui contredisent ce que remarque Littré : « Contrairement à ce qu'avance Littré, c'est en combinaison avec de qu'il [aucun] s'emploie surtout de nos jours [...]. La langue populaire, chose curieuse, est sur ce point d'accord avec celle des lettrés: «Y en a d'aucuns qui vous diront que...»H. Monnier (cité dans Nyrop)». Ils remarquent (Ibid., 217) que d'aucun(e)s "peut s'employer ainsi [d'aucuns au pluriel] aujourd'hui encore. Mais il y faut quelques précautions ». Grevisse (1993: 1080) avance que d'aucuns peut encore s'utiliser au pluriel avec son ancien sens positif de «quelques-uns, certains » «dans la langue écrite soignée, mais aussi dans la langue parlée de certaines régions (notamment de l'Orléanais)». Buridant (2000: 179) note également que l'on trouve d'aucuns «encore, en français contemporain : d'aucuns dans d'aucuns disent, etc. ».

${ }^{17}$ Nous avons relevé 23 mots insérés au XIII ${ }^{\mathrm{e}}$ siècle, 42 au XIV ${ }^{\mathrm{e}}$ siècle, 43 au XV ${ }^{\mathrm{e}}$ siècle, 44 au XVI ${ }^{\mathrm{e}}$ siècle, 63 au XVII ${ }^{\mathrm{e}}$ siècle, 28 au XVIII ${ }^{\mathrm{e}}$ siècle, 42 au $\mathrm{XIX}^{\mathrm{e}}$ siècle et 48 au $\mathrm{XX}^{\mathrm{e}}$ siècle.

${ }^{18}$ Notons que de nombreux adjectifs comme beau, gros, petit, léger, doux, fou, noble, sage, vilain, etc., ne comptant que quelques attestations pour chacun, peuvent s'insérer dans le SN aucun $\mathrm{N}$ : 18 adjectifs au XIII ${ }^{\mathrm{e}}$ siècle, 36 au XIV ${ }^{\mathrm{e}}$ siècle, $44 \mathrm{au} \mathrm{XV}^{\mathrm{e}}$ siècle, $40 \mathrm{au} \mathrm{XVI}{ }^{\mathrm{e}}$ siècle, 57 au XVII ${ }^{\mathrm{e}}$ siècle, 27 au XVIII ${ }^{\mathrm{e}}$ siècle, 35 au $\mathrm{XIX}^{\mathrm{e}}$ siècle et 41 au $\mathrm{XX}^{\mathrm{e}}$ siècle. 
${ }^{19}$ Les constructions directes de aucun se font principalement avec l'indéfini autre et d'une façon moindre avec les adjectifs juste, galant, envieux, inutile, impur, méchant, bon, beau, mauvais...

${ }^{20}$ Aucun peut se joindre indirectement via la préposition de uniquement à des adjectifs (rare, meilleur, bon, arrêté, fixe, formel, réel, véritable, heureux, fixe, nécessaire, utile, gros, galant, joli, aimable...). Notons la régression de ces constructions indirectes au cours du XIX ${ }^{\mathrm{e}}$ siècle (10 attestations) que nous trouvons employées avec les adjectifs bon, comparable, efficace, essentiel, fort, inutile, net, pire, positif et urgent. Cette régression se confirme au cours du $\mathrm{XX}^{\mathrm{e}}$ siècle (6 attestations). Les dernières constructions indirectes se trouvent utilisées avec les adjectifs digne, extraordinaire, inutile précis, riche et salubre.

${ }^{21}$ La majorité des exemples rencontrés dans notre corpus sont calqués sur le modèle des phrases données par Féraud (1787: 204) nécessitant la présence de en. A côté de ces emplois, nous avons trouvé également quelques exemples où aucun est représentant de certains noms (chevaliers, leçons, mauvais ministres, mots...), ce qui équivaut à l'emploi de en.

${ }^{22}$ On dirait que, lorsque aucun a pris une valeur négative, l'expression d'aucuns s'est figée pour dénoter sans ambiguïté le sens d'indéfini positif. Le figement est visible dans les exemples où d'aucuns peut suivre des prépositions (...̀̀/par d'aucuns...) ou apparaître comme objet direct (...punir d'aucuns qui...) ou comme sujet (D'aucuns qui...). Dans ce cas, comme la préposition de, dans d'aucuns, ne joue plus son rôle de préposition, on pourrait parler d'une lexicalisation de l'expression. 